\title{
Oxygen and sulphur abundances of solar-type stars of the solar-neighborhood
}

\author{
G. F. Porto de Mello ${ }^{1}$, F. Requeijo ${ }^{2}$, L. da Silva ${ }^{2}$, M. Kürster ${ }^{3}$, \\ S. Els ${ }^{4}$, and M. Endl ${ }^{5}$
}

${ }^{1}$ Observatório do Valongo/UFRJ, Ladeira do Pedro Antônio 43, 20080-090 Rio de Janeiro, RJ, Brazil (email: gustavo@ov.ufrj.br), ${ }^{2}$ Observatório Nacional/MCT, Brazil, ${ }^{3}$ Heidelberg University, Germany, ${ }^{4} \mathrm{CTIO} / \mathrm{Chile},{ }^{5}$ McDonald Observatory, USA

\begin{abstract}
The Sun has typical abundances of both oxygen and sulphur for its metallicity, age and galactic orbit, when compared to the nearby solar-type stars. This result favors a solution of the old riddle of the solar overabundance in oxygen with respect to the local interstellar medium as caused by the recent infall of metal-poor gas over the disk, rather than the competing explanations of an outward migration of the Sun from a inner, and metal richer, birthplace in the disk, or a last-minute supernova which might have enriched the proto-solar nebula.
\end{abstract}

Keywords. Galaxy: abundances, stars: abundances, ISM: abundances.

\section{Introduction}

The oxygen abundance of the interstellar medium, $\log \mathrm{N}(\mathrm{O})=8.50$ (Meyer et al. 1998) is $40 \%$ down from solar, $\log \mathrm{N}(\mathrm{O})=8.69$ (Allende-Prieto et al. 2001) for all lines of sight. HII regions and B-type stars show the same deficiency (Daflon et al. 2001). Carbon, krypton, nitrogen and, possibly, sulphur are also deficient in the ISM (Fitzpatrick \& Spitzer 1997, Cardelli \& Meyer 1997). Grain depletion might explain part of the oxygen and carbon deficiency, but krypton, a noble gas, offers no such alternative explanation, and sulphur has probably a high gas-grain phase ratio (Harris \& Mas Hesse 1986). Three hypothesis were put forward to explain this discrepancy. First, a last minute SNII might have enriched the protosolar nebula in $\alpha$-elements. Elements owed to SNII should then be overabundant in the ISM, a pattern challenged by sulphur, albeit with large uncertainty; and the ISM should have solar abundances of the elements not owed to SNII, but carbon and nitrogen are also deficient. Second, an infall of metal poor gas over the disk might have diluted the ISM. The observed deficiency of almost all elements favors this (Snow \& Witt 1996), but sulphur apparently breaks this pattern. Third, the Sun might have migrated from an inner and more metal-rich location in the Galaxy (Wielen et al. 1996): yet dynamical migration cannot explain all observed abundances dispersions (François \& Matteucci 1993). The Sun, in this case, should be anomalously rich in $\alpha$-elements. Our goal is to choose among these three scenarios by deriving homogeneous oxygen and sulphur abundances, both products of SNII, of a sample of solar-type stars spanning a wide range of metallicities, ages and galactic orbits.

\section{Results}

We employ for oxygen the $\lambda 7774$ lines (OPD/LNA/Brazil, $\mathrm{R}=20,000$, ESO/FEROS, $\mathrm{R}=47,000)$ and the forbidden $\lambda 6300$ line (ESO/CES, $\mathrm{R}=210,000$ ); for sulphur, the $\lambda 8695$ line $(\mathrm{OPD} / \mathrm{LNA}, \mathrm{R}=30,000, \mathrm{ESO} / \mathrm{FEROS})$ : the $\mathrm{S} / \mathrm{N}$ was superior to 200 in 


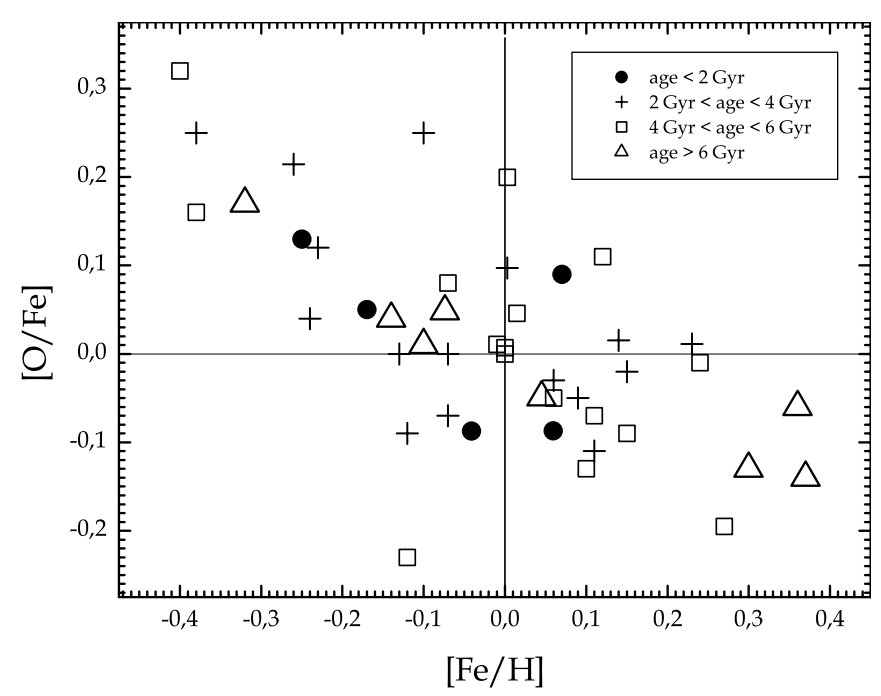

Figure 1. Oxygen abundances: the stars are divided into three age groups.

all cases. The analysis was differential with respect to the Sun, using MARCS model atmospheres and atmospheric parameters taken from the literature. Even though there is some heterogeneity in the sources of the atmospheric parameters, great care was taken to select parameters from authors employing similar methods. This, and the homogeneity of our spectra, should guarantee good internal consistency in the abundance results. We analysed the oxygen and sulphur abundances stratifying the stars into different groups, according to their isochronal ages (fig. 1: when a reasonable age solution was allowed in theoretical HR diagrams) and their mean galactocentric distances. The Sun was found to be typical in its $[\mathrm{O} / \mathrm{Fe}]$ and $[\mathrm{S} / \mathrm{Fe}]$ abundances for its metallicity, age and galactic orbit: this result makes the last minute SNII and the dynamical migration hypotheses untenable. A recent dilution of the ISM by the impact of a $10^{6} \mathrm{M}_{\odot}$ cloud, originating Gould's Belt, has been suggested as the source of the ISM deficiency (Comerón \& Torra 1994). The ISM abundances beyond Gould's Belt are more similar to solar, in good agreement with this proposal (André et al. 2003). Thus, dilution of the local ISM by metal-poor gas is the favored explanation for its deficiency with respect to the local population of solar-type stars. The apparent absence of an ISM deficiency for sulphur should be verified with data of greater accuracy.

\section{References}

Allende-Prieto, C., Lambert, D.L. \& Asplund, M. 2003, ApJ 556, L63

André, M.K., Oliveira, C.M., Howk, J.C., Ferlet, R., Désert, J.M., Hébrard, G., Lacour, S., Lecavelier des Étangs, A., Vidal-Madjar, A. \& Moos, H.M. 2003, ApJ 591, 100

Cardelli, J.A. \& Meyer, D.M. 1997, ApJ 477, L67

Comerón, F. \& Torra, J. 1994 A $\& A$ 281, 35

Daflon, S., Cunha, K., Becker, S.R. \& Smith, V.V. 2001 ApJ 552, 307

Fitzpatrick, E.L. \& Spitzer, L.J. 1997 ApJ 475, 623

François, P. \& Matteucci, F. 1993, A\&̋A 280, 136

Harris, A.W. \& Mas Hesse, J.M. 1986, ApJ 308, 240

Meyer, D.M., Jura, M. \& Cardelli, J.A. 1998, ApJ 493, 222

Snow, T.P. \& Witt, A.N. 1996, ApJ 468, L65

Wielen, R., Fuchs, B. \& Dettbarn, C. 1996, A\&A 314, 438 